\title{
Metastatic Cervical Adenocarcinoma
}

National Cancer Institute

\section{Source}

National Cancer Institute. Metastatic Cervical Adenocarcinoma. NCI Thesaurus. Code C153389.

A cervical adenocarcinoma that has spread to another anatomic site. 